\title{
Clinical Value of Nipple Discharge and Serum Markers of Breast Cancer
}

\author{
Yun'ai Liang ${ }^{1}$, Zongling $\mathrm{Mou}^{2}$, Jie Zhang ${ }^{3}$, Xin $\mathrm{Li}^{4}$, \\ Ying Chen ${ }^{5,6} \&$ Gangping Wang ${ }^{1, *}$ \\ ${ }^{1}$ Department of Pathology, Rizhao People's Hospital, Rizhao, 276826, China \\ 2 Department of General Surgery, Rizhao People's Hospital, Rizhao, 276826, China \\ ${ }^{3}$ Department of Gynaecology, Rizhao People's Hospital, Rizhao, 276826, China \\ ${ }^{4}$ Department of Chinese Medicine, , Rizhao People's Hospital, Rizhao, 276826, China \\ ${ }^{5}$ Department of Immunology, Qiingdao University, Qiingdao, 266071, China \\ ${ }^{6}$ Department of Laboratory, Rizhao People's Hospital, Rizhao, 276826, China \\ *Corresponding author: Gangping Wang;,E-mail: wgprzph93@126.com
}

Keywords: Breast cancer; biomarker; nipple discharge; serum; pathology; diagnosis.

\begin{abstract}
Serum biomarkers are of diagnostic value and can be used for follow-up and prognostic factors. However, serum protein biomarkers show limited diagnostic sensitivity and specificity in stand-alone assays breast cancer because their levels refect tumor burden. In this study, we reviewed the levels of CA15-3, TSGF, CA125 and CEA both in nipple discharge and serum in 179 cases of breast lesions to assess the clinical value of nipple discharge and serum tumor markers in diagnosis, follow-up and prognostic in breast cancer. Our results indicate that the nipple discharge and serum levels of CA15-3, TSGF, CA125 and CEA in breast carcinomas patients were significantly higher than those in the benign disease $(P<0.01)$. Aditionally, The levels of the four tumor markers in nipple discharge were significantly higher than in the serum $(P<0.01)$. The levels of the four biomarkers in nipple discharge had positive correlation with histological grade, clinical stage, the Ki-67 index, expression of VEGF and HER-2/neu, lymphnode metastas and tumor recurrence $(P<0.05$, respectively), and negative correlation with the level of ER or $\mathrm{PR}(P<0.05$, respectively). The sensitivity of the four serum tumor markes in combination was only $69.77 \%$, in contrast, the combined detection both in discharge and serum was $97.67 \%$, and the negative predictive value was $99.03 \%$. The sensitivity of combined detection both in nipple discharge and serum were significantly higher than other detection $(P<0.05)$. The four tumor markers in nipple discharge are as novel biomarker in dignosis and judging the prognosis of breast cancer. The dynamic combined detection of the four tumor markers both in nipple discharge and serum are helpful to the stratification of preoperative patients and benefit to better prewarning markers for monitoring their recurrence and metastasis of tumors in clinic, but cannot increase the sensitivity of judging the patients with early breast cancer.
\end{abstract}

\section{Introduction}

Breast cancer is a major public health problem throughout the world [1]. It is the most common cancer among women both in developed and developing countries. Nipple discharge is a relatively common breast complaint accounting for up to $5 \%$ of which women seek medical advice [2-4]. Majority of nipple discharge comes forth spontaneously and has a pathological outcome. Of the patients presented with nipple discharge, the incidence of malignancy is reported as a range from 
$5 \%$ to $21 \%$ [5-6]. A large portion of patients with nonpuerperal nipple discharge contain one or several symptoms including: spontaneous unilateral, serous and bloody discharge [7]. In this study, we reviewed the levels of carbohydrate antigen15-3 (CA15-3), tumor specific growth factor (TSGF), carbohydrate antigen 125 (CA125) and carcino embryonic antigen (CEA) both in nipple discharge and serum in 179 cases of breast lesions and their relationship with biological parameters were studied and analyzed retrospectively in order to study the Clinical and pathological value, especially in early stage of breast cancer.

\section{Materials and methods}

\section{Patients}

Permission was obtained from the Rizhao people's Hospital Ethical Committee to collect breast tissues and all the patients signed informed consents. One hundred and seventy-nine cases of breast lesions including breast ductal carcinoma $(n=43)$ and benign lesions $(n=136)$ were collected during excision surgery at Rizhao people's Hospital. The diagnosis was determined according to the current World Health Organization classification system (WHO 2012) [1]. The biological parameters beseides the express of estrogen receptor (ER), progesterone receptor (PR), human epidermal growth factor receptor-2 (HER-2/neu), vascular endothelial growth factor (VEGF) and Ki-67 detected by immunohistochemistry Ultra Sensitive ${ }^{T M} S-P$ Kit (Maixin-Bio, China) method according to the manufacturer's instructions and the levels of CA15-3, TSGF, CA125 and CEA measured in serum and nipple discharge with Electrochemiluminescence method and their relationship were studied. Tissue samples were fixed in $10 \%$ neutral buffered formalin and embedded in paraffin. Tissue sections were deparaffinized and rehydrated using standard procedures. Immunoreactions signals were visualized using the DAB substrate, which stains the target protein yellow. Cell membrane reactivity for the HER-2 oncoprotein was evaluated following a similar approach and the mean value was used to score each case. Tumors expressing HER-2 in $>10 \%$ of the cancer cells were considered as positive. In addition, for ER, PR and Ki-67 expression the percentage of cancer cells showing a nuclear reactivity was recorded after inspection of all optical fields at $200 \times$ and the mean value was used to score each case. Tumors with expression of $>10 \%$ (not $1 \%$ ) of cancer cells were considered to be positive for ER and PR .

\section{Biomarkers measurement of serum and nipple discharge samples}

We performed biomarkers of blood serum and nipple discharge analysis in this study. For CA15-3, TSGF, CA125 and CEA analysis, $3 \mathrm{ml}$ heparinized blood and $0.5 \mathrm{ml}$ of nipple discharge was drawn from each individual. The biomarkers were detected with electroc- hemiluminescence method in the clinical laboratory in Rizhao People's Hospital, and was compared with 30 cases of normal pregnant women. The cut off values of CA15-3, TSGF, CA125 and CEA in serum are 25.00U/ml, 70.00U/ml, $35.00 \mathrm{U} / \mathrm{ml}$ and $3.40 \mathrm{ng} / \mathrm{ml}$, and those of nipple discharge are $35.00 \mathrm{U} / \mathrm{ml}, 95.00 \mathrm{U} / \mathrm{ml}, 40.00 \mathrm{U} / \mathrm{ml}$ and $9.8 \mathrm{ng} / \mathrm{ml}$.

\section{Statistical analysis}

SPSS ver 17. 0 statistical software was used to detect the data, and the results were expressed with $(\bar{x} \pm s)$. Measurement data between groups was compared with $t$ test, while enumeration data with $\chi^{2}$ test. The $P$ value was considered to be significant if less than 0.05 .

\section{Results}




\section{Diagnostic Value of Nipple Discharge and Serum Biomarkers}

One hundred and seventy-nine cases presented nipple discharge and the Clinical information of nipple discharge lesion was summarized in Table 1. Thirty-four cases $(18.99 \%, 34 / 179)$ presented skin changes, such as skin color change $(n=10)$, skin orange-peel-texture $(n=13)$ and breast skin ulceration $(n=11)$. There was difference between color of discharge $(P=0.01)$ and discharge duct amount $(P=0.01)$.

Table 1 The Clinical information of nipple discharge lesion $(n=179)$

\begin{tabular}{lllll}
\hline & Benign & Malignant & $\chi^{2}$ Value & $P$ Value \\
\hline $\begin{array}{l}\text { location } \\
\text { left } \\
\text { right }\end{array}$ & 76 & 30 & 2.61 & 0.11 \\
\hline $\begin{array}{l}\text { Color of discharge } \\
\text { bloody }\end{array}$ & 60 & 13 & & \\
$\quad$ non-bloody & 49 & 25 & 6.59 & 0.01 \\
\hline size & 87 & 18 & & \\
$\leq 2 \mathrm{~cm}$ & & & & \\
$>2 \mathrm{~cm}$ & 78 & 27 & 0.40 & \\
\hline Duct amount & 58 & 16 & & 0.01 \\
single-duct & 75 & 12 & 0.70 & \\
mult-duct & 61 & 31 & & \\
\hline
\end{tabular}

The nipple discharge and serum levels of CA15-3, TSGF, CA125 and CEA were shown in Table 2. The nipple discharge and serum levels of the four biomarkers in malignant lesion were significantly higher than those in benign lesion patients $(P<0.01)$. The levels of the four biomarkers in nipple discharge were significantly higher than those in the serum $(P<0.01)$. The sensitivity of the four serum tumor markes in combination was only $69.77 \%$,in contrast, the dynamic combined detection both in discharge and serum was $97.67 \%$, and the negative predictive value was $99.03 \%$. The sensitivity of combined detection both in nipple discharge and serum were significantly higher than other detection $(P<0.05)$.

Table 2 The biomarkers in malignant and benign lesion patients $(n=179)$

\begin{tabular}{cccccc}
\hline Subgroup & $\mathrm{n}$ & CA15-3 $(\mathrm{U} / \mathrm{ml})$ & TSGF $(\mathrm{U} / \mathrm{ml})$ & CA125 $(\mathrm{U} / \mathrm{ml})$ & CEA $(\mathrm{ng} / \mathrm{ml})$ \\
\hline $\begin{array}{c}\text { Nipple discharge } \\
\text { Malignant }\end{array}$ & 43 & $128.21 \pm 28.63$ & $212.42 \pm 45.71$ & $115.71 \pm 41.08$ & $109.23 \pm 30.94$ \\
Benign & 136 & $25.13 \pm 6.14^{\mathrm{a}}$ & $67.01 \pm 14.98^{\mathrm{a}}$ & $29.41 \pm 7.22^{\mathrm{a}}$ & $7.46 \pm 1.75^{\mathrm{a}}$ \\
\hline Serum & & & & & \\
Malignant & 43 & $93.79 \pm 21.80$ & $145.46 \pm 32.11$ & $86.68 \pm 21.37$ & $43.29 \pm 16.81$ \\
Benign & 136 & $18.61 \pm 3.98^{\mathrm{b}}$ & $56.01 \pm 11.99^{\mathrm{b}}$ & $20.29 \pm 4.60^{\mathrm{b}}$ & $2.40 \pm 0.72^{\mathrm{b}}$ \\
\hline
\end{tabular}

Biomarkers level of nipple discharge compared with benign lesion, ${ }^{a} P<0.01$; Serum level compared with benign group, ${ }^{\mathrm{b}} P<0.01$.

\section{Nipple Discharge Biomarker Level Relation with Clinicopathological Factors}

The relationship of the four marker levels of discharge in breast cancer patients and the different clinical pathological factors were shown in Figure 1. The levels of the four biomarkers in nipple 
discharge had a positive correlation with the Ki-67 value index, histological grade, clinical stage, expression of VEGF and HER-2/neu, lymphnode metastas, and tumor recurrence $(P<0.05$, respectively), and negative correlation with the level of ER or $\operatorname{PR}(P<0.05$, respectively), but there was no correlation with BMI, age at dignosis, menarche or menopause ( $P>0.05$, respectively).

a

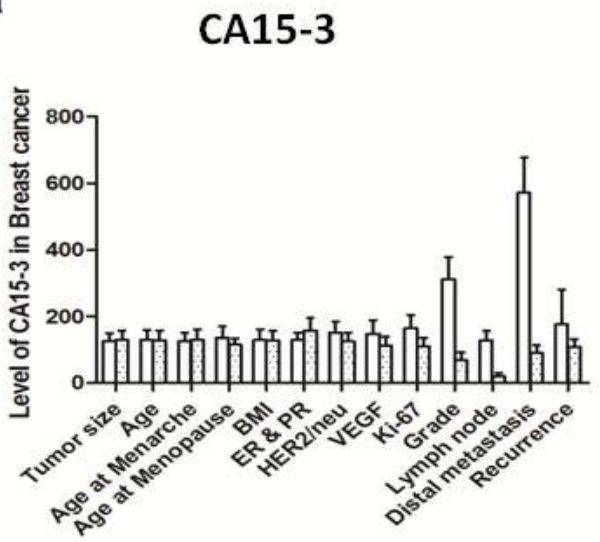

C

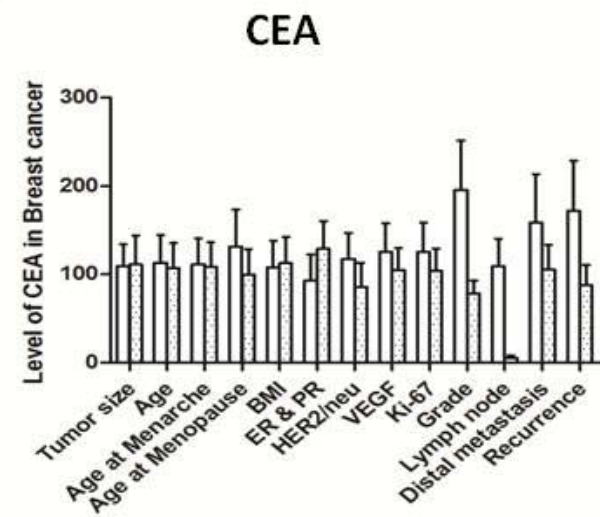

b

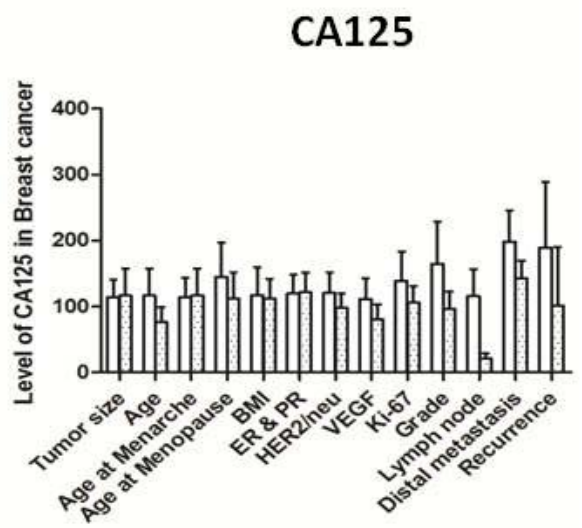

d

TSGF

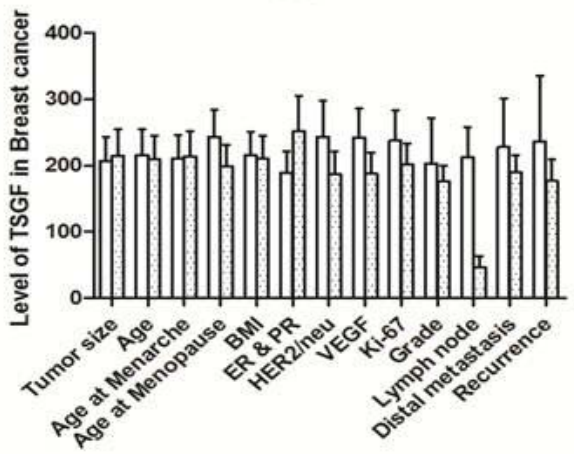

Figure 1 Nipple discharge biomarker level of CA15-3 (A), CA125 (B), CEA (C) and TSGF (D) relation with clinicopathological factors in breast cancer.

\section{Discussion}

Breast cancer its morbidity is on the rise year by year in the world. The discharge from nipple is common, accounting for $5 \%$ of all breast related symptoms [8]. Majority of nipple discharge comes forth spontaneously and has a pathological outcome. It locates in or originates from mammary ducts and generally harbors a benign or malignant breast tumor [2-5]. Abnormal nipple discharge is most commonly caused by benign conditions like intraductal papillomas, duct ectasia, papillomatosis, mastitis, fibrocystic changes [6-9]. The incidence of breast cancer in patients presenting with abnormal nipple discharge is between $5 \%$ to $21 \%$ [2,3,10]. A majority of patients with breast cancer who manifest with isolated nipple discharge have an early stage disease associated with ductal carcinoma in situ. Discharge owing to ductal carcinoma in situ has been shown to be a marker for extensive disease, which often requires mastectomy to achieve adequate surgical margins $[11,12]$. Discharge can be an early sign of breast cancer $[9,12]$. Diffusely spreading intraductal carcinomas which often have no clinically palpable breast mass can manifest as pathological discharge. The 
tumor-related indicators in all individuals cannot be detected systemically and comprehensively due to limited economics and techniques, thereby, combined detection of specifc tumor markers is of great importance $[13,14]$. CEA is useful for diagnosis of recurrence and prognosis of breast cancer $[15,16]$. CA15-3 was used in the management of prognosis, metastasis and recurrence in breast cancer patients [17,18]. Preoperative level of CEA and CA15-3 in serum were well known as a significant effect on prognosis of breast cancer [19,20]. Cancer antigen 125 (CA125) was found to be up-regulated in breast cancer tissues and not expressed in non-neoplastic ducts [21]. In this study, the nipple discharge and serum levels of the four biomarkers in malignant lesion were significantly higher than those in benign lesion patients $(P<0.01)$. The levels of the four biomarkers in nipple discharge were significantly higher than those in the serum $(P<0.01)$. Furthermore, our previous research shown CA15-3, TSGF, CA125 and CEA also had a certain clinical signifcance in the early diagnosis of tumors $[2,4]$. In this study, the sensitivity of the four serum tumor markes in combination in diagnosis of breast cancer was only $69.77 \%$, in contrast, the dynamic combined detection both in discharge and serum was $97.67 \%$, and the negative predictive value was $99.03 \%$. The dynamic combined detection of the four tumor markers both in nipple discharge and serum are benefit to early diagnosis and interference and better prewarning markers for monitoring their recurrence and metastasis of breast cancer, but cannot increase the sensitivity of diagnosis of Precancerous lesions. In this study, we analyze for the first time the ER, PR, HER-2, Ki-67 and the combined detecting CA15-3, TSGF, CA125 and CEA simultaneously in the same sample both in discharge and serum, and achieved a better application effect. The results in the study and our previous research [2,4,13-14] revealed that breast cancer tumor size, onset age, menarche and menopause age had no signifcant association with nipple discharge level of the 4 biomarkers in nipple discharge among breast cancer groups. Based on body mass index (BMI), females were categorized into two groups, below $30 \mathrm{~kg} / \mathrm{m}^{2}$ and above or equal $30 \mathrm{~kg} / \mathrm{m}^{2}$. No signifcant associations were found between the four biomarkers in nipple discharge level and BMI categories. The nipple discharge levels of the 4 biomarkers were markedly higher in those ER and PR negative patients compared to those positive patients. There were a significantly difference of the level between HER-2/neu positive patients and HER-2/neu negative patients, Ki-67 proliferation index $\leq 14 \%$ patients and $>14 \%$ patients. Furthermore,with increase in pathological staging, levels of the four biomarkers in serum and nipple discharge gradually increased. In addition, the levels of serum and nipple discharge were significantly increased in those patients with lymph node metastasis and distal metastasis compared to those patients without metastasis metastasis. Values with distal metastasis were notably higher than with region lymph node metastasis. The postoperative follow-up results revealed that levels of nipple discharge and serum CA15-3, TSGF, CA125 and CEA in recurrence group were obviously higher than in non-recurrence group. The nipple discharge levels of the CA15-3, TSGF, CA125 and CEA four tumor markers in recurrence group were obviously higher than in non-recurrence group.

\section{Conclusion}

The study results suggest that combined detection of the indicators above can judge the prognosis better, which is of great importance to monitor recurrence and metastasis. Besides, combined detection of CA15-3, TSGF, CA125 and CEA can improve their own sensitivity without decreasing diagnostic accuracy and specifcity of breast cancer, but the effect is still limited. Our results suggest that the tumor markers of CA15-3, TSGF, CA125 and CEA selected in the current study, single or in a panel, do not predict the presence of a very early stage of breast cancer. It cannot be excluded 
that the proteins may predict better in combinations with other proteins not selected here. Future studies should therefore preferentially select a broader target set of potential biomarkers.

\section{Competing interests}

The authors declare that they have no competing interests.

\section{Acknowledgements}

This study was financially supported by Project of Application Technology Research and Development Project Foundation in Rizhao (2014SZSH02), Medicine and Health Care Science and Technology Development Plan Project Foundation of Shandong Province (2014WS0282 and 2014WSA11003), Medical Staff' Science and Technology Innovation Projects of Shandong Province Medical Union Committee (201515), as well as Scientific Research Key Projects of Jining Medical University (JY2013KJ051).

\section{References}

[1] S.R. Lakhani, I.O. Ellis, S.J. Schnitt, P.H. Tan and M.J. Vijver. WHO classification of tumours of the breast [M]. Lyon, France: IARC Press, 2012.

[2] G. Wang, Y. Qin, J. Zhang, J. Zhao, Y. Liang, Z. Zhang, et al. Nipple Discharge of CA15-3, CA125, CEA and TSGF as a New Biomarker Panel for Breast Cancer. Int. J. Mol. Sci. 2014;15: 9546-9565.

[3] H.I. Vargas, L. Romero and R.T. Chlebowski. Management of bloody nipple discharge. Current treatment options in oncology. 2002;3 (2):157-161.

[4] G. Wang, S. Yang and H. Zhang. The clinical significance of survivin and vascular endothelial growth factor in breast cancer and precancerous lesions. J Investig Med. 2015;63 (8 Suppl):S24.

[5] I. Montroni, D. Santini, G. Zucchini, M. Fiacchi, S. Zanotti, G. Ugolini, et al. Nipple discharge: is its significance as a risk factor for breast cancer fully understood? Observational study including 915 consecutive patients who underwent selective duct excision. Breast cancer research and treatment.16 2010;123 (3):895-900.

[6] E.R. Sauter, A. Klein-Szanto, B. Macgibbon and H. Ehya. Nipple aspirate fluid and ductoscopy to detect breast cancer. Diagnostic cytopathology. 2010;38 (4):244-251.

[7] C. Furuya, H. Kawano, T. Yamanouchi, A. Oga, J. Ueda and M. Takahashi. Combined evaluation of $\mathrm{CK} 5 / 6$, ER, p63, and MUC3 for distinguishing breast intraductal papilloma from ductal carcinoma in situ. Pathology international. 2012; 62 (6): 381-390.

[8] J.E. Lang and H.M. Kuerer. Breast ductal secretions: clinical features, potential uses, and possible applications. Cancer control. 2007,14: 350-359.

[9] S. Zervoudis, G. Iatrakis, P. Economides, D. Polyzos and I. Navrozoglou. Nipple discharge screening. Womens Health. 2010,6: 135-151.

[10]Dolan RT, Butler JS, Kell MR, T.F. Gorey and M.A. Stokes . Nipple discharge and the efficacy of duct cytology in evaluating breast cancer risk [J]. Surgeon, 2010, 8: 252-258. 
[11]V. Parthasarathy and U. Rathnam.Nipple discharge: an early warning sign of breast cancer [J]. Int J Prey Med, 2012, 3: 810-814.

[12]L. Chen, W.B. Zhou, Y. Zhao, X.A. Liu ,Q. Ding, X.M. Zha and S. Wang. Bloody nipple discharge is a predictor of breast cancer risk: a meta-analysis [J]. Breast Cancer Res Treat, 2012, 132: 9-14.

[13] G.P. Wang, M.H.Qin, Y.A. Liang, H. Zhang and Z.F. Zhang. The significance of biomarkers in nipple discharge and serum in diagnosis of breast cance. In: Advanced Engineering and Technology. Liquan Xie, Dianjian Huang, (eds.). 2014, CRC Press, pp665-671.

[14]W. Gang-ping, M. Jing and C. Ying. The expressions of HIF-1 $\alpha$ and COX-2 in tissue and their relationship with the level of tsgf, CA15-3 and neoadjuvant chemosensitivity in breast cancer patients. J Investig Med. 2015;63 (8 Suppl):S70-71.

[15]Y. Shao, X. Sun, Y. He, C. Liu and H. Liu. Elevated Levels of Serum Tumor Markers CEA and CA15-3 Are Prognostic Parameters for Different Molecular Subtypes of Breast Cancer. PloS one. 2015; 10 (7):e0133830.

[16]P. Stieber, D. Nagel, I. Blankenburg, V. Heinemann, M. Untch, I. Bauerfeind, et al. Diagnostic efficacy of CA 15-3 and CEA in the early detection of metastatic breast cancer-A retrospective analysis of kinetics on 743 breast cancer patients. Clinica chimica acta; international journal of clinical chemistry. 2015;448:228-231.

[17]M.J. Kim, B.W. Park, J.B. Lim, H.S. Kim, J.Y. Kwak, S.J. Kim, et al. Axillary lymph node metastasis: CA-15-3 and carcinoembryonic antigen concentrations in fine-needle aspirates for preoperative diagnosis inpatients with breast cancer. Radiology. 2010;254 (3):691-697.

[18]M.J. Duffy, D. Evoy and E.W. McDermott. CA 15-3: uses and limitation as a biomarker for breast cancer. Clinica chimica acta; international journal of clinical chemistry. 2010; 411 (23-24): 1869- 1874.

[19]J.S. Lee, S. Park, J.M. Park, J.H. Cho, S.I. Kim and B.W. Park. Elevated levels of preoperative CA 15-3 and CEA serum levels have independently poor prognostic significance in breast cancer. Annals of oncology: official journal of the European Society for Medical Oncology/ESMO. 2013; 24 (5): 1225-1231.

[20]S.G. Wu, Z.Y. He, J. Zhou, J.Y. Sun, F.Y. Li, Q. Lin, et al. Serum levels of CEA and CA15-3 in different molecular subtypes and prognostic value in Chinese breast cancer. Breast. 2014; 23 (1): 88- 93.

[21]I. Lakshmanan, M.P. Ponnusamy, S. Das, S. Chakraborty, D. Haridas, P. Mukhopadhyay, et al. MUC16 induced rapid G2/M transition via interactions with JAK2 for increased proliferation and anti-apoptosis 43 in breast cancer cells. Oncogene. 2012;31 (7):805-817. 